\title{
RECONSTRUCTION OF 3D TUBULAR STRUCTURES FROM CONE-BEAM PROJECTIONS
}

\author{
Jia $\mathrm{Li}^{*}$ \\ Dept. of Elect. and Comp. Engrg. \\ Oakland University \\ Rochester, MI 48309, USA
}

\author{
Laurent Cohen
}

\author{
CEREMADE \\ University Paris - Dauphine \\ 75775 Paris cedex 16, France
}

\begin{abstract}
A new method of reconstructing three-dimensional (3D) tubular structures from cone-beam projections is proposed in this paper. Minimal path method is applied to detect 2D tubular structures in the cone-beam projections. The extracted $2 \mathrm{D}$ information are fused to design a weight function over the 3D volume so that minimal path method can be applied again to extract centerlines of 3D tubular structures. The validity of the proposed weight function relies on the sparsity of 3D images containing tubular structures. The procedure of estimating the radius of $3 \mathrm{D}$ tube from $2 \mathrm{D}$ projections is also described. The proposed method requires neither cone-beam reconstruction, nor tree matching, one of which is usually necessary in the existing reconstruction methods. The method is robust to small motions between different projections.
\end{abstract}

Index Terms - Tubular structures, cone-beam, minimal path

\section{INTRODUCTION}

Reconstruction of three dimensional (3D) tubular structures from multiple cone-beam projections has important applications in medical image analysis. For example, the extraction of 3D coronary arteries in the analysis of X-ray coronary angiographies [1], and the intracerebral vascular reconstruction in neurosurgeon practice [2], are both concerned with reconstructing 3D blood vessels from conebeam projections. Using a digital flat panel, cone-beam projections are usually obtained in a rotational acquisition mode with a fixed step in rotational angle. A few projections can be selected from the whole sequence for the reconstruction task. For example, in coronary arteries reconstruction, the projections corresponding to the same cardiac time are selected such that a static $3 \mathrm{D}$ reconstruction is possible. Motion estimation can be performed after the static reconstructions at different cardiac time are obtained. We are mainly concerned with static reconstruction from a few projections in this paper, so the issue of motion estimation will not be discussed, although it is one of the main tasks in quantitative coronary analysis.

The existing reconstruction methods can be classified into two categories according to whether it requires $3 \mathrm{D}$ volume reconstruction or not. Methods in the first category usually perform conebeam image reconstruction, then apply 3D segmentation to extract the tubular structures [3]. For general 3D cone-beam reconstruction, most common non-iterative algorithms are based on the backprojection formula proposed by Feldkamp [4]. Although filtered back-projection reconstruction is faster than iterative reconstruction, the resulted images usually contain sever artifacts due to the over simplified line-integral model. Iterative cone-beam reconstructions

\footnotetext{
*The author performed the work while taking sabbatical leave at CEREMADE in the University of Paris - Dauphine in 2010.
}

claim better reconstruction quality, but suffer from high computational cost. After 3D volume is reconstructed, tubular structures can be extracted using minimal path method [5]. Li and Yezzi proposed to represent $3 \mathrm{D}$ tubular structure as a 4D curve to incorporate radius information such that a global minimizing 4D path can be a complete solution to the reconstruction task [6]. The methods that do not require $3 \mathrm{D}$ cone-beam reconstruction usually first perform $2 \mathrm{D}$ segmentation in each projection to extract $2 \mathrm{D}$ centerlines, then perform tree matching over the trees from different projections to reconstruct 3D tubular structures. For example, in [2], Bullitt et al addressed the specific problem of reconstruction of $3 \mathrm{D}$ curves from a pair of $2 \mathrm{D}$ curves in the presence of error. In [1], Blondel et al performed multiocular matching to build correspondences between the centerlines of different projections. The approach developed by Jandt's group also falls into this category [7]. When processing 2D projections, each pixel is assigned a rank to indicate its likelihood of being inside a blood vessel. Then $2 \mathrm{D}$ ranking functions of different viewing angles are paired to form a weight function of the volume that is used in $3 \mathrm{D}$ centerline extraction. However, the issue of radius estimation is not addressed in Jandt's work.

In this paper, we present a reconstruction method, which extracts both the centerlines and radii of 3D tubular structures. The method does not require cone-beam reconstruction, so it belongs to the second category discussed above. $2 \mathrm{D}$ centerlines in each projection are first extracted using minimal path method. Then the distance map corresponding to the distance between pixels and 2D centerlines is computed for each projection. A 3D weight function is obtained by fusing the distance maps of all the projections. Finally 3D centerlines are reconstructed from the $3 \mathrm{D}$ weight function via minimal path method, and radii at discrete centerline points are estimated from the size of 2D tubular structures. Our contribution to the field is the introduction of a new weight function for minimal path segmentation, which exploits the sparsity of tubular structure images, as well as a low cost radius estimation procedure.

The remainder of this paper is organized as follows. In Section 2, minimal path method and tubular structure detection based on optimally oriented flux are briefly reviewed. Section 3 details the 3D weight function design and radius estimation. In Section 4 , the experimental results with simulated coronary data sets are presented and discussed. We conclude the paper in Section 5.

\section{MINIMAL PATHS AND TUBULAR STRUCTURE DETECTION}

\subsection{Minimal Path}

Minimal path, also called geodesic, is a path connecting a starting point and an ending point that minimizes the total cost accumulated along the path. Let $\gamma:[0,1] \rightarrow \Omega$ be a smooth curve, the cost 
functional can be regarded as a weighted length of $\gamma$ and expressed as

$$
L(\gamma)=\int_{0}^{1} W(\gamma(t))\left\|\gamma^{\prime}(t)\right\| \mathrm{d} t
$$

where $\gamma^{\prime}(t)$ is the derivative of $\gamma$, and $W$ is a weight function defined on the domain $\Omega$ that can vary with applications. Using this setting, the solution to the minimal path problem $\gamma^{*}$ is a global minimizer of the weighted length,

$$
\gamma^{*}=\underset{\gamma \in P\left(x_{s}, x_{e}\right)}{\operatorname{argmin}} L(\gamma)
$$

where $x_{s}$ and $x_{e}$ represent the starting and ending points of the path, and $P\left(x_{s}, x_{e}\right)$ is the set of all the paths between $x_{s}$ and $x_{e}$.

In computer vision community, minimal path method has been applied to image segmentation, especially the centerline extractions of tubular structures, such as roads and blood vessels. For this kind of application, weight function is usually designed such that the region inside the tubular structures has a small value relative to the background or other objects. Therefore the optimizer prefers the path passing through tubular structures to the solutions that pass through background.

To solve minimal path problem, minimal distance map associated with the starting point $x_{s}$ must be computed. The minimal distance map $U_{x_{s}}(x)$ is a function over the image domain $\Omega$, whose value equals the weighted length of the minimal path connecting points $x_{s}$ and $x$, i.e.

$$
U_{x_{s}}(x)=\min _{\gamma \in P\left(x_{s}, x\right)} L(\gamma)
$$

where $P\left(x_{s}, x\right)$ is the set containing all the possible paths between $x_{s}$ and $x$, and $x \in \Omega$. $U_{x_{s}}(x)$ satisfies Eikonal equation $\nabla U_{x_{s}}=$ $W$, which can be solved by different schemes after discretization. An efficient non-iterative algorithm for solving the equation is fast marching algorithm [8]. We have applied fast marching algorithm to compute minimal distance map in both the 2D and 3D centerline extractions. Once the minimal distance map is computed, minimal path can be obtained by gradient descent starting from the ending point $x_{e}$. Fig. 1 shows a 2D cone-beam projection and the 2D centerlines extracted from it.

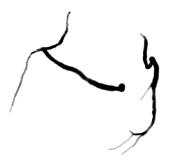

(a) A cone-beam projection

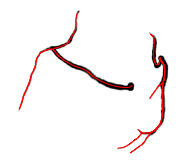

(b) Extracted 2D centerlines
Fig. 1. A cone-beam projection and the associated 2D centerlines $\gamma^{2 D}$.

\subsection{Tubular Structure Detection}

When applying minimal path method to solve image segmentation problem, the difficult task is to design a weight function $W$ such that the desired features can be represented by the minimizing curve. In case of tubular structure segmentation, the weight function $W$ should incorporate the information from tubular structure detection. Such kind of detection aims at detecting the presence of tubular structures as well as estimating local orientation and size of the structure. The detectors that have been proposed include Hessian based filters and optimally oriented flux (OOF). Hessian based filters have difficulties when there are other objects adjacent to the tubular structure. To overcome this drawback, Law and Chung introduced OOF, which estimated the structure orientation by finding a projection axis on which the projected gradient flux is minimized [9]. The advantage of OOF is that its performance is not disturbed by the adjacent objects. In [6], Li and Yezzi proposed to represent a tubular surface as the envelope of a family of spheres with continuously changing center points and radii, and developed two different 4D weight functions which can ensure the sphere with the desired radius has lower weight than those spheres with inaccurate radii. The $2 \mathrm{D}$ centerline extraction in our approach has adopted a 2D version of Li and Yezzi's method, which allows simultaneous extraction of centerline and radius. A drawback of the method is that it requires user inputs of start points and end points of tubular structures. The key point detection technique [10] can be incorporated to remove the requirement of end points, but hasn't been implemented by this time.

\section{FROM 2D CONE-BEAM PROJECTIONS TO 3D WEIGHT FUNCTION}

The first step in our approach is to process each cone-beam projection to extract 2D centerlines and estimate local thickness of the projected tubular structures. After that, 3D centerline reconstruction and radius estimation are done sequentially. As mentioned in Section 1 , our weight function for $3 \mathrm{D}$ centerline extraction is computed from centerlines in $2 \mathrm{D}$ projections. So the focus of this section is to design a $3 \mathrm{D}$ weight function using the extracted $2 \mathrm{D}$ information. Table 1 lists the notations that will be used in our discussion. Fig. 2 illustrates the physical meanings of these notations.

Table 1. Notations

\begin{tabular}{ll} 
& \multicolumn{1}{c}{ Table 1. Notations } \\
\hline \hline Notations & Descriptions \\
\hline$x$ & a point in the 3D field of view \\
$S_{i}$ & radiation source location of the $i$-th \\
& projection \\
$\operatorname{proj}_{i}$ & the $i$-th projection operator \\
$\gamma^{3 D}$ & centerlines of the 3D tubular structure \\
$\gamma_{i}^{2 D}$ & 2D centerlines in the $i$-th projection, \\
& equivalent to proj $\left(\gamma^{3 D}\right)$ \\
$d_{\gamma_{i}^{2 D}}$ & Euclidean distance map associated with \\
& $\gamma_{i}^{2 D}$, defined over the $i$-th projection \\
\hline \hline
\end{tabular}

\subsection{Weight Function Design}

Minimal path method relies on proper design of weight function to obtain meaningful solution. Based on the information extracted from $2 \mathrm{D}$ projections, we are interested in forming a $3 \mathrm{D}$ weight function suitable for minimal path method to extract centerlines of 3D tubular structures. Due to the nature of minimal path, the desired weight function should have small values inside the tubular structure, and lowest value on the centerlines. 


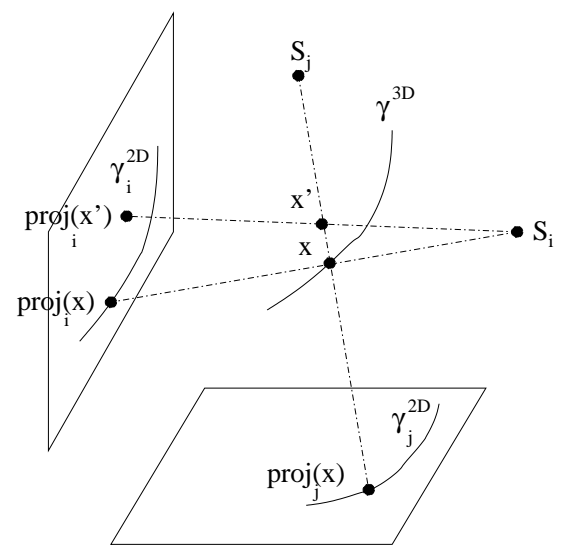

Fig. 2. Illustrations of notations.

For each 2D projection image, we first compute the distance map associated with the extracted centerlines. Using the $i$-th projection as an example, the map is defined as a pixel's Euclidean distance to $\gamma_{i}^{2 D}$, i.e.

$$
d_{\gamma_{i}^{2 D}}\left(\operatorname{proj}_{i}(x)\right)=\min _{y \in \gamma_{i}^{2 D}}\left\|\operatorname{proj}_{i}(x)-y\right\|,
$$

where $\operatorname{proj}_{i}$ represents the $i$-th projection operator, and $\gamma_{i}^{2 D}$ is the $2 \mathrm{D}$ centerline extracted from the $i$-th projection.

Based on the distance function $d_{\gamma_{i}^{2 D}}$, we propose a weight function

$$
W(x)=\max _{i}\left(d_{\gamma_{i}^{2 D}}\left(\operatorname{proj}_{i}(x)\right)\right),
$$

for $3 \mathrm{D}$ centerline extraction. We claim that $W(x)$ has the following properties:

1. $\forall x, W(x) \geq 0$.

2. $\forall x \in \gamma^{3 D}, W(x)=0$.

3. The set $X_{0}=\left\{x: x \notin \gamma^{3 D}\right.$ and $\left.W(x)=0\right\}$ is very small if the $3 \mathrm{D}$ volume containing tubular structures is sparse.

By sparse image, we refer to images where background pixels/voxels are dominant. For example, in the X-ray coronary angiography, the pixels in vessel regions are about $5-10 \%$ of all the pixels in a $2 \mathrm{D}$ projection. In terms of the $3 \mathrm{D}$ volume to be reconstructed, the voxels in vessel regions are about $0.04-0.1 \%$ of the total number of voxels.

\section{Proof}

1. This property is inherited from the nature of the distance function $d_{\gamma_{i}^{2 D}}$.

2. $x \in \gamma^{3 D} \Rightarrow \forall i, \operatorname{proj}_{i}(x) \in \gamma_{i}^{2 D} \Rightarrow \forall i, d_{\gamma_{i}^{2 D}}\left(\operatorname{proj}_{i}(x)\right)=0$ $\Rightarrow W(x)=0$.

3. $W(x)=0$ implies that $\operatorname{proj}_{i}(x) \in \gamma_{i}^{2 D}, \forall i$. If $x \notin \gamma^{3 D}$, $\operatorname{proj}_{i}(x) \in \gamma_{i}^{2 D}, \forall i$ is an event that is unlikely to occur due to the sparsity of the image, especially when the number of projections used in reconstruction is larger than 2. For a point $x \notin \gamma^{3 D}$, the event $\operatorname{proj}_{i}(x)$ belongs to $\gamma_{i}^{2 D}$ occurs if and only if $x$ belongs to the surface formed by $S_{i}$ and $\gamma^{3 D}$. We can assume the probability of this event is less than a small constant $c$ for all $i$ due to the sparsity of the volume. The value of $c$ varies with the level of sparsity. In

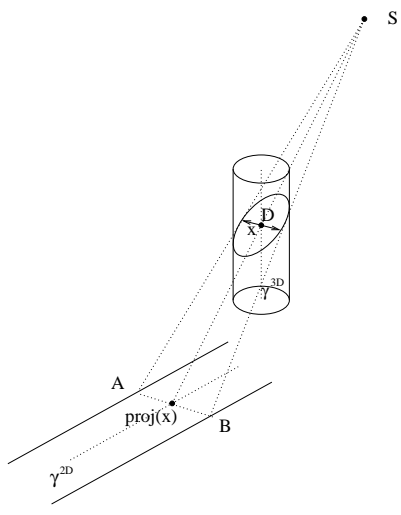

Fig. 3. Estimation of 3D tubular structure's radius.

simulated data that we have tested, $c$ is less than 0.1. It's also reasonable to assume that the event that $\operatorname{proj}_{i}(x)$ belongs to $\gamma_{i}^{2 D}$ is independent to the event that $\operatorname{proj}_{j}(x)$ belongs to $\gamma_{j}^{2 D}$, for $i \neq j$. Let $K$ be the number of projections used in reconstruction, the probability that $\operatorname{proj}_{i}(x) \in \gamma_{i}^{2 D}, \forall i$ or $W(x)=0$ is less than $c^{K}$. In other words, the size of the set $X_{0}=\left\{x: x \notin \gamma^{3 D}\right.$ and $\left.W(x)=0\right\}$ is less than $N_{x} \cdot N_{y} \cdot N_{z} \cdot c^{K}$, where $N_{x}, N_{y}$ and $N_{z}$ are the size of the three dimensions of the volume.

We use Fig. 2 to illustrate the argument above. Fig. 2 shows two 3D points, $x \in \gamma^{3 D}$ and $x^{\prime} \notin \gamma^{3 D}$. Because $x^{\prime}$ is in the line formed by $S_{j}$ and $x, \operatorname{proj}_{j}\left(x^{\prime}\right)$ equals $\operatorname{proj}_{j}(x)$, and belongs to $\gamma_{j}^{2 D}$, which implies $d_{\gamma_{j}^{2 D}}\left(\operatorname{proj}_{j}\left(x^{\prime}\right)\right)=0$. However, due to the sparsity, $x^{\prime}$ is not in any line formed by $S_{i}$ and a point in $\gamma^{3 D}$. So $\operatorname{proj}_{i}\left(x^{\prime}\right)$ does not belong to $\gamma_{i}^{2 D}$, which implies $d_{\gamma_{i}^{2 D}}\left(\operatorname{proj}_{i}\left(x^{\prime}\right)\right)>0$ and $W\left(x^{\prime}\right)>0$.

These properties of $W(x)$ can assure that it achieves minimum value along $\gamma^{3 D}$ and larger values out of $\gamma^{3 D}$, which subsequently assures the accurate extraction of 3D centerlines of the tubular structures in sparse images. The existence of the set $X_{0}$ usually does not have an impact to the 3D centerline extraction because $X_{0} \notin \gamma^{3 D}$. As long as the starting point provided by the user is in $\gamma^{3 D}$, the points in $X_{0}$ will not be extracted by the minimal path solver. In real practice, a small positive constant $\epsilon$ is added to $W(x)$ to prevent loops in the path.

\subsection{Radius Estimation}

We estimate the radii of 3D tubular structure from the radii extracted in $2 \mathrm{D}$ projections. Fig. 3 shows the general relationship between the thickness of the 3D tubular structure and the radius of its $2 \mathrm{D}$ projections. $S$ is the location of radiation source. For a point $x$ in the $3 \mathrm{D}$ centerline $\gamma^{3 D}$, its projection $\operatorname{proj}(x)$ is on the $2 \mathrm{D}$ centerline $\gamma^{2 D}$. A and $B$ are two boundary points that form a segment passing through $\operatorname{proj}(x)$ and perpendicular to $\gamma^{2 D}$. The three points, $S, A$ and $B$, define a plane that intersects the $3 \mathrm{D}$ tube.

Let the radius of the tube at $x$ be $r$. The distance $d(S, x)$ is usually much larger than the value of $r$. So line $S A$ and line $S B$ bounding the tube at $x$ are near parallel. Under the assumption of parallelism, the shortest distance between the two lines at $x$, represented by $D$, can be regarded as the diameter of the tube. The value of $D$ can be calculated from the location of $S, x, A$ and $B$. If the 


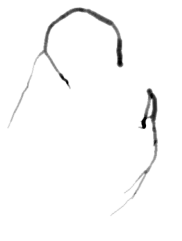

(a) Projection 1

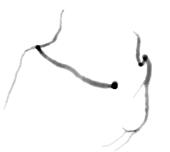

(c) Projection 3

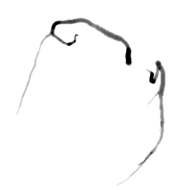

(b) Projection 2

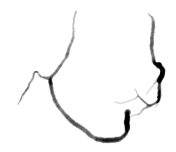

(d) Projection 4

Fig. 4. Cone-beam projections used in reconstruction.

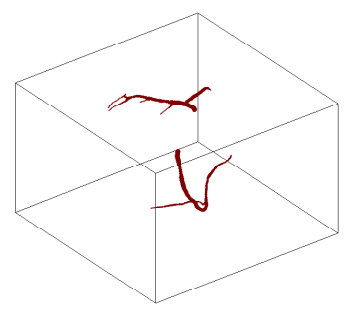

(a) Simulated coronary arteries

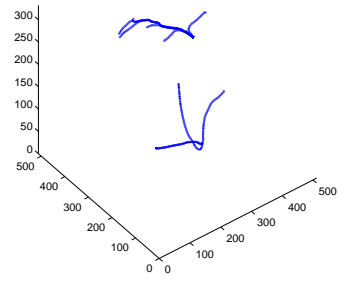

(b) Extracted centerlines

Fig. 5. Reconstruction of simulated 3D coronary trees.

assumption of parallelism doesn't hold, $r$ can still be estimated via a general procedure, which involves a little bit more computation.

\section{RESULTS}

We present the results of tubular structure reconstruction using the proposed method in this section. The method has been tested over simulated data to quantify the accuracy of the results.

To generate the simulated data, blood vessels were constructed from binary 3D coronary trees and embedded in a $512 \times 512 \times 332$ volume image. Fig. 5 (a) shows the simulated coronary arteries. The volume image was projected to produce a series of cone-beam projections of size $800 \times 800$ to simulate rotational angiography. The projection operator is a distance driven operator. Four projections, with rotational angle at $0^{\circ}, 30^{\circ}, 60^{\circ}$ and $90^{\circ}$, have been selected as the input to the reconstruction algorithm. They are shown in Fig. 4. After extracting the $2 \mathrm{D}$ centerlines in these projections, the $3 \mathrm{D}$ weight function is computed and fed to minimal path method to extract 3D centerlines. The result is shown in Fig. 5 (b).

We quantify the accuracy of minimal path solution using a sim-

ple metric. Let $\gamma^{3 D}=\left\{p_{1}, p_{2}, \ldots, p_{M}\right\}$ be the true centerline and $\gamma^{*}=\left\{v_{1}, v_{2}, \ldots, v_{K}\right\}$ be the minimal path solution. For each point $v_{i} \in \gamma^{*}$, we find its two nearest neighbors in $\gamma^{3 D}$ and compute the distance between $v_{i}$ and the line formed by the two nearest neighbors. The resulted distance is regarded as the error of $\gamma^{*}$ at $v_{i}$. The error of $\gamma^{*}$ is obtained by taking average of this error over the whole curve. It should be noted that curve smoothness, which is also an important factor in fidelity quantification, cannot be measured with this metric.

To test the robustness of the proposed method, random translations have been added to the vertical direction of the four projections to simulate the motion caused by imperfect synchronization in cardiac time. A standard Gaussian was sampled and multiplied with different values of $\sigma$ to generate different levels of random motions. Table 2 shows a snapshot of such generated motions in vertical direction. Applying the listed motions, we got five minimal path solutions corresponding to $\sigma=0,0.5,1,2,4$, separately. The errors of these solutions are recorded at the bottom of Table 2. Fig. 6 compares one of the 3D centerlines extracted in the experiment of $\sigma=4$ with the ground truth. As indicated in Table 2, the error of the minimal path solution equals 1.9186 pixels when $\sigma=4$. These results demonstrate that the proposed method is not very sensitive to small motions in vertical direction.

Table 2. Simulated different levels of random motions and reconstruction errors in pixels.

\begin{tabular}{c|c|c|c|c|c}
\hline \hline & \multicolumn{5}{|c}{ Motions in vertical direction (pixel) } \\
\cline { 2 - 6 } & $\sigma=0$ & $\sigma=0.5$ & $\sigma=1$ & $\sigma=2$ & $\sigma=4$ \\
\hline Proj 1 & 0 & -0.5732 & 0.3273 & -1.1766 & 4.2671 \\
Proj 2 & 0 & 0.5955 & 0.1746 & 4.3664 & 0.2371 \\
Proj 3 & 0 & 0.5946 & -0.1867 & -0.2728 & -0.3826 \\
Proj 4 & 0 & -0.0188 & 0.7258 & 0.2729 & -3.3294 \\
\hline Error & 0.9954 & 1.2906 & 1.2866 & 1.3623 & 1.9186 \\
\hline \hline
\end{tabular}

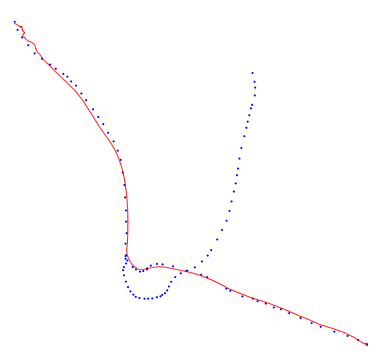

Fig. 6. One of the 3D centerlines extracted when $\sigma=4$. The blue dots represent the ground truth, while the red line is the minimal path solution.

\section{CONCLUSION}

In this paper, a new method for static reconstruction of 3D tubular structures from cone-beam projections has been presented. The sparsity of tubular structures is exploited to design a $3 \mathrm{D}$ weight function 
for the extraction of centerlines by minimal path method. The feasibility of the method has been evaluated over simulated data sets. The results show that the method can achieve fairly good accuracy when the cone-beam projections are disturbed by small motions in the vertical direction. Current version of the method requires both start points and end points of the tubular structure as inputs. In the future, a minimal path method with key point detection technique can be implemented to remove the requirement for end points.

\section{REFERENCES}

[1] Christophe Blondel, Grégoire Malandain, Régis Vaillant, and Nicholas Ayache, "Reconstruction of coronary arteries from a single rotational x-ray projection sequence," IEEE Trans. on Medical Imaging, vol. 25, no. 5, pp. 653-663, 2006.

[2] E. Bullitt, A. Liu, and S. M. Pizer, "Three-dimensional reconstruction of curves from pairs of projection views in the presence of error. I. Algorithms," Med. Phys., vol. 24, no. 11, pp. 1671-1678, 1997.

[3] Meihua Li, Haiquan Yang, and Hiroyuki Kudo, "An accurate iterative reconstruction algorithm for sparse objects: application to 3D blood vessel reconstruction from a limited number of projections," Phys. Med. Biol., vol. 47, pp. 2599-2609, 2002.

[4] L.A. Feldkamp, L.C. Davis, and J.W. Kress, "Practical conebeam algorithm," J. Opt. Soc. Am. A, vol. 1, no. 6, pp. 612-619, 1984.

[5] L.D. Cohen and T. Deschamps, "Segmentation of 3D tubular objects with adaptive front propagation and minimal tree extraction for 3D medical imaging," Comput. Methods Biomech. Biomed. Eng., vol. 10, no. 4, pp. 289-305, 2007.

[6] Hua Li and Anthony Yezzi, "Vessels as 4-D curves: Global minimal 4-D paths to extract 3-D tubular surfaces and centerlines," IEEE Trans. on Medical Imaging, vol. 26, no. 9, pp. 1213-1223, 2007.

[7] Uwe Jandt, Dirk Schäfer, Michael Grass, and Volker Rasche, "Automatic generation of 3D coronary artery centerlines using rotational x-ray angiography," Med. Image Anal., vol. 13, pp. 846-858, 2009.

[8] J. A. Sethian, "A fast marching level set method for monotonically advancing fronts," Proc. of the National Academy of Sciences, vol. 93, no. 4, pp. 1591-1595, 1996.

[9] Max W. Law and Albert C. Chung, "Three dimensional curvilinear structure detection using optimally oriented flux," in ECCV 2008: LNCS 5305, Berlin Heidelberg, 2008, pp. 368382, Springer-Verlag.

[10] Fethallah Benmansour and Laurent D. Cohen, "Fast object segmentation by growing minimal paths from a single point on 2D or 3D images," Journal of Mathematical Imaging and Vision, 2008. 